\title{
Effectiveness of a website and mobile phone based physical activity and nutrition intervention for middle-aged males: Trial protocol and baseline findings of the ManUp Study
}

Mitch J Duncan ${ }^{1 *}$, Corneel Vandelanotte ${ }^{1}$, Richard R Rosenkranz², Cristina M Caperchione ${ }^{3}$, Hang Ding ${ }^{4}$, Marcus Ellison ${ }^{1}$, Emma S George ${ }^{5}$, Cindy Hooker ${ }^{1}$, Mohan Karunanithi ${ }^{4}$, Gregory S Kolt ${ }^{5}$, Anthony Maeder ${ }^{6}$, Manny Noakes ${ }^{7}$, Rhys Tague ${ }^{6}$, Pennie Taylor ${ }^{7}$, Pierre Viljoen ${ }^{8}$ and W Kerry Mummery ${ }^{9}$

\begin{abstract}
Background: Compared to females, males experience higher rates of chronic disease and mortality, yet few health promotion initiatives are specifically aimed at men. Therefore, the aim of the ManUp Study is to examine the effectiveness of an IT-based intervention to increase the physical activity and nutrition behaviour and literacy in middle-aged males (aged 35-54 years).

Method/Design: The study design was a two-arm randomised controlled trial, having an IT-based (applying website and mobile phones) and a print-based intervention arm, to deliver intervention materials and to promote self-monitoring of physical activity and nutrition behaviours. Participants $(n=317)$ were randomised on a 2:1 ratio in favour of the IT-based intervention arm. Both intervention arms completed assessments at baseline, 3, and 9 months. All participants completed self-report assessments of physical activity, sitting time, nutrition behaviours, physical activity and nutrition literacy, perceived health status and socio-demographic characteristics. A randomly selected sub-sample in the IT-based $(n=61)$ and print-based $(n=30)$ intervention arms completed objective measures of height, weight, waist circumference, and physical activity as measured by accelerometer (Actigraph GT3X). The average age of participants in the IT-based and print-based intervention arm was 44.2 and 43.8 years respectively. The majority of participants were employed in professional occupations (IT-based $57.6 \%$, Print-based 54.2\%) and were overweight or obese (IT-based 90.8\%, Print-based 87.3\%). At baseline a lower proportion of participants in the IT-based (70.2\%) group agreed that 30 minutes of physical activity each day is enough to improve health compared to the print-based (82.3\%) group $(p=.026)$. The IT-based group consumed a significantly lower number of serves of red meat in the previous week, compared to the print-based group $(p=.017)$. No other significant between-group differences were observed at baseline.

(Continued on next page)
\end{abstract}

\footnotetext{
* Correspondence: m.duncan@cqu.edu.au

${ }^{1}$ Central Queensland University, Institute for Health and Social Science Research, Centre for Physical Activity Studies, Bruce Highway, Rockhampton, QLD 4700, Australia

Full list of author information is available at the end of the article
} 
(Continued from previous page)

Discussion: The ManUp Study will examine the effectiveness of an IT-based approach to improve physical activity and nutrition behaviour and literacy. Study outcomes will provide much needed information on the efficacy of this approach in middle aged males, which is important due to the large proportions of males at risk, and the potential reach of IT-based interventions.

Trial registration: ACTRN12611000081910

Keywords: Physical activity, Nutrition, Males, Website, Internet, Online, Mobile phone, Self-monitoring, Literacy, Intervention

\section{Background}

Reducing disease and the prevalence of avoidable risk factors associated with poor health in males is attracting considerable attention within the public health agenda. This is evidenced in both developed and developing countries by the advancement of several recent policy initiatives and other strategies dedicated to improving the health of males $[1,2]$. These initiatives are based on a recognition that, compared to females, males experience higher rates of overall premature mortality, cardiovascular disease related mortality, suicide, diabetes, and obesity $[2,3]$. These health outcomes are linked to males' high levels of behavioural risk factors including high levels of physical inactivity, poor nutrition behaviours, high risk drinking and smoking $[2,3]$.

Improving the physical activity levels of males is vital to improving their health, as physical inactivity is one of the most significant risk factors for the leading causes of preventable death and burden of disease in Australian males [2]. Physical inactivity is characterized by high levels of sedentary behaviours, such as sitting, and/or low levels of participation in moderate-to-vigorous intensity physical activity, such as walking. Australian surveillance data indicate that males report more than 3 hours of occupational sitting, and 5.5 hours of total sitting per day $[4,5]$. Although approximately $53 \%$ of males report engaging in sufficient levels of physical activity for health benefit, the proportion of males engaging in sufficient physical activity has not increased in recent years [6].

Improving nutrition behaviours is similarly important for improving male health, as poor nutrition behaviours also rank highly among risk factors for the leading causes of preventable death and disease in Australian males [2]. Poor nutrition behaviours are characterized by low consumption of fruits, fibre, vegetables, and fish, and high consumption of saturated fat and alcohol. Fruit and vegetable consumption is low in males, with fewer than $6.5 \%$ of males consuming recommended daily serves of fruit and vegetables [7,8] and 15\% reporting that they consumed alcohol at high-risk levels [9]. Furthermore, approximately half of adult males consume full-cream milk on a usual basis and takeaway food at least once per week, which is indicative of high saturated fat intake [10].

Despite ample evidence of demonstrated need for improvement in health and related behavioural risk factors, males are frequently underrepresented in healthpromotion and chronic disease prevention intervention efforts [11,12], and report that the interventions that are frequently adopted by females do not appeal to them [13]. Thus, there is a need to develop interventions that specifically target physical activity and nutrition behaviours of males in a way that is tailored to their preferences. The format of the messages, presentation of information delivered, mode of information delivery, and methods to engage participants are key intervention strategies than can be tailored to males. Website-delivered interventions can be tailored to specific user preferences and needs, are viewed favourably by males, and can positively alter levels of overweight, physical activity, and selected dietary behaviours $[12,14,15]$. The accessibility of such websitedelivered interventions may also be more appealing to males who cannot attend face-to-face interventions due to clashes with work, family responsibilities, and other schedules [16]. Additionally the capacity of website-delivered interventions to be accessed by large numbers of males in need of health behaviour change is beneficial. The provision of educational materials, goal setting strategies, and tools to promote self-monitoring have been shown to be effective methods to change physical activity and nutrition behaviours [12,17-19]. Our formative research on these issues demonstrated that males are somewhat knowledgeable on the levels of physical activity and healthy eating required to promote health, but that they also want information on these topics provided in a clear and simple way to avoid confusion associated with multiple media campaigns and messages [20,21]. Tailoring health promotion messages in this way is important as the materials not only need to provide information in an 'easily digestible' format for males, but must also empower males to use this information to positively change their behaviours [22]. This process of applying knowledge to change behaviour, or health literacy, plays an important role in health promotion and is relatively low in many populations [22]. Males also acknowledge the importance 
of self-monitoring behaviours, but want this to be completed in a time-efficient and user-friendly manner [21]. As such, website-delivered interventions that incorporate these characteristics and features may be useful to positively change physical activity and nutrition behaviours.

Self-monitoring of health behaviour is a common behaviour change strategy implemented in many health promotion interventions [23], and is positively associated with greater behaviour change across a range of health behaviours $[17,18]$. Mobile (cell) phones that store data locally and/or connect to the internet offer participants a modern and technologically sophisticated way to selfmonitor behaviour, may offer greater convenience when compared to more traditional self-monitoring methods, and allow transfer of data to a website that contains other intervention materials and components [21]. However, the use of websites and/or mobile phones, to self-monitor physical activity or nutrition behaviours, specifically targeted at males, remains largely untested. This manuscript describes the rationale, design, and baseline findings from the ManUp Study, which aims to examine the effectiveness of an information technology (IT) based intervention that uses both websites and mobile phones to improve the physical activity and nutrition behaviour and literacy in middle-aged males.

\section{Methods/Design}

The ManUp Study design is a two-arm randomised controlled trial with assessment points at baseline, three, and nine months. One intervention arm used an ITbased approach (website and mobile phones) to deliver physical activity and healthy eating promotion materials, and to promote self-monitoring of these behaviours. The second intervention arm used a more conventional print-based approach to deliver the same health promotion materials. Both intervention arms provided participants with the same materials and ability to self-monitor their behaviours, although the IT-based intervention additionally provided participants with automated feedback on their progress towards completing physical activity and nutrition goals, and the ability to interact with other participants on the website platform as a social support mechanism (Table 1). The IT-based intervention allowed participants to self-monitor physical activity and nutrition behaviours using either the website or mobile phone platform. A print-based comparison group was selected rather than a wait-list control group as it has been demonstrated that print-based interventions are effective $[24,25]$. Participants provided informed consent prior to participation and the study was approved by Central Queensland University, and the University of Western Sydney's Human Research Ethics Committees. The study was registered with the Australian New Zealand Clinical Trials Registry (ACTRN12611000081910).

\section{Participants, recruitment and group allocation}

Eligible participants were males aged 35 to 54 years old who (1) owned a mobile telephone, (2) had access to the internet, (3) did not have a mobility impairment, (4) resided in the cities of Gladstone or Rockhampton (Queensland, Australia), and (5) were classified as low risk to commence an exercise regime [26]. Participants $(\mathrm{n}=317)$ were recruited using a variety of techniques including advertisements via local newspapers, trading magazines, face-to-face information sessions with local businesses, and distribution of leaflets and posters to local businesses, medical clinics, and offices of allied health professionals. Rolling participant recruitment occurred from October 2010 to September 2011. Following initial screening for inclusion criteria, participants were randomly allocated to an intervention arm. Group assignment was conducted on a two-to-one ratio in favour of the IT-based intervention arm. Unequal group allocation was conducted to maximize the number of participants allocated to the intervention arm that is less frequently examined in male populations [19]. Randomization lists were generated using freely available software (www.randomization.com). Participants were blinded to group allocation until baseline assessments were completed.

\section{ManUp intervention}

The study was labeled "ManUp" to have men identify with the intervention and also to challenge men to take responsibility for their health. Similarities and differences in intervention components between the IT and print groups are summarized in Table 1 and reflected the ability of the IT-based intervention to include components related to automated feedback and participant interaction in an online environment. The ManUp intervention was designed on the basis of our review of physical activity interventions in males [12], formative research that identified the enablers and barriers that males faced in engaging in more physical activity and healthy eating [20] and the specific requirements for the IT-based components suggested by males [21].

Both intervention arms were designed to engage participants in making healthy changes to their physical activity and nutrition behaviours, and improve their health literacy about these behaviours. This was achieved by the provision of educational materials, increasing the frequency of participation in physical activity and healthy eating by the completion of a series of "challenges", and self-monitoring progress towards the completion of each challenge. Participants were able to access their group's intervention materials throughout the nine-month study period. Educational materials were specifically designed to be clear and uncomplicated in the presentation of the benefits of physical activity and healthy eating, the amount or type required to achieve 
Table 1 Components of the ManUp intervention

\begin{tabular}{|c|c|c|c|c|}
\hline Major Component & Sub-component & Description & $\begin{array}{l}\text { IT-based } \\
\text { Intervention } \\
\text { Arm }\end{array}$ & $\begin{array}{l}\text { Print-based } \\
\text { Intervention } \\
\text { Arm }\end{array}$ \\
\hline
\end{tabular}

\section{Educational}

Materials

\section{Physical Activity}

What is physical

activity

Benefits of physical

activity

Where to be active

Getting started

Further information

\section{Healthy Eating}

What is healthy eating

Benefits of healthy eating

Where to eat healthily

Getting started

Further information

\section{Body Weight}

What is a healthy body weight

Benefits of a healthy

body weight

How to achieve a healthy body weight

Further information
Description of

- What is 'physical activity'

- The different intensities of physical activity

- The physical activity guidelines for Australian adults Summary of the health, social and economic benefits of physical activity participation

Summary of the locations to be physically active in the study area, including parks, community facilities, commercial recreation centres.

Summary of the steps people should do to minimise risk when commencing a physical activity regime, including link to pre-exercise screening tool. Includes information on selecting the correct equipment for the activity, warming up, stretching, cooling down, progressively increasing activity, hydration and sun safety.

URLs of other health promotion websites and resources for further information on physical activity

Description of

- What is 'healthy eating'

- Where appropriate a serving size is defined

- The healthy eating guidelines for Australian adults

Summary of the health benefits of healthy eating

Summary of the outlets that provide fresh food options in the study area

Summary of strategies to make choosing healthy eating a part of daily routines

URL addresses of other health promotion websites and resources for further information on healthy eating

Description of how a healthy body weight is defined using BMI and waist circumference

Summary of the health benefits of maintaining a healthy body weight

Summary of the strategies that can be used to maintain and/or achieve a healthy body weight

URL addresses of other health promotion websites and resources to assist in maintaining and/or achieving a healthy body weight

- Ability to record progress towards completing any of the challenges

- Ability to record body weight, height and waist circumference 


\begin{tabular}{lll}
\hline & - Automatically generated summary of all data \\
& recorded & \\
& - Ability to schedule an activity and receive \\
& text or email reminder \\
& - Visual summary of progress towards \\
& completing ManUp challenges \\
Social-support & - Ability to view 'mates' progress \\
& - Ability to comment on a the profile page of \\
& a mate \\
ManUp challenges & - Ability to complete group based challenges \\
& Light, Mid and Full Strength Physical Activity \\
& and Healthy Eating Challenges
\end{tabular}

health benefit, and how to achieve the amounts required for health benefits $[12,20,21]$. Educational materials were also designed to encourage males to change their health behaviours by recognizing how physical inactivity and poor nutrition can adversely affect health and by using this recognition as a stimulus to set goals to make positive changes to these behaviours [27]. The ManUp physical activity and healthy eating "challenges" were informed by Social Cognitive Theory and Self Regulation Theory, and developed to change target behaviours by having participants engage in goal setting and selfmonitoring behaviours based on these challenges $[28,29]$. Goal setting and self-monitoring was operationalised by having participants select a challenge and record their progress towards completing the challenge.

\section{ManUp physical activity and healthy eating challenges} The "ManUp challenges" varied in duration and the amount of activity or healthy eating that males were asked to achieve. The challenges were designed to increase the overall levels of physical activity and healthy eating, rather than achieving any particular guideline for the behaviours. Variation in the duration and requirements for each challenge are described below. The challenge concept was adopted in ManUp based on our formative research $[12,20,21]$. Challenges were constructed to provide participants with specific, measureable, and time-based goals to achieve, which is consistent with established goal setting strategies [30], by specifying the weekly and total volume of activity across the duration of the challenge.

In total, six ManUp physical activity challenges (see Table 2) and a single ManUp healthy eating challenge were provided for participants to select from. For each challenge, participants could select from three different 'strengths' to participate in: light strength (three weeks), mid strength (six weeks) and full strength (12 weeks). The different strength challenges also varied in the weekly volume of physical activity and healthy eating to be completed and could be completed in any order. ManUp physical activity challenges included the activities of walking, cycling, swimming,

Table 2 Description of the ManUp physical activity and healthy eating challenges

\begin{tabular}{llll}
\hline Activity & Light Strength (3 weeks) & Mid Strength (6 weeks) & Full strength (12 weeks) \\
\hline Walking & $1.5 \mathrm{hrs} /$ week or & $2.5 \mathrm{hrs} /$ week or & $3.5 \mathrm{hrs} /$ week or \\
& 7500 steps/day & $10000 \mathrm{steps} /$ day & $12000 \mathrm{steps} / \mathrm{day}$ \\
Cycling & $1 \mathrm{hr} /$ week or & $2 \mathrm{hrs} /$ week & $4 \mathrm{hrs} /$ week \\
& $25 \mathrm{~km} /$ week & or $50 \mathrm{~km} /$ week & or $100 \mathrm{~km} /$ week \\
Swimming & $0.5 \mathrm{hr} /$ week or & $1 \mathrm{hr} /$ week or & $1.5 \mathrm{hrs} /$ week or \\
& $1 \mathrm{~km} /$ week & $2 \mathrm{~km} /$ week & $3 \mathrm{~km} /$ week \\
Running & $0.5 \mathrm{hr} /$ week or & $1 \mathrm{hr} /$ week or & $2.0 \mathrm{hrs} /$ week or \\
Sport \& Recreation & $5 \mathrm{~km} /$ week & $10 \mathrm{~km} /$ week & $20 \mathrm{~km} /$ week \\
Strengthening & $0.5 \mathrm{hr} /$ week & $1 \mathrm{hr} /$ week & $1.5 \mathrm{hrs} /$ week \\
& Set 8 exercises & Set 8 exercises & Set 8 exercises \\
& $1 \times$ set (8-10 reps) & $2 \times$ set (8-10 reps) & $3 \times$ set (8-10 reps) \\
Healthy Eating & $2 \times /$ week & $2 \times /$ week & $2 \times /$ week
\end{tabular}


running, strengthening, sport and recreation, and were selected based on recreation activities that Australian males frequently participate in [31]. The strengthening challenge included any resistance-based exercises such as free weights, machine weights, and body weight exercises. The sport and recreation challenge included any team-based activity or individual activity (i.e. soccer, football or groupbased fitness class) not covered by the remaining five physical activity challenges.

The ManUp healthy eating challenges were based on achieving a number of daily healthy eating goals: in total there were ten healthy eating goals that could be achieved. The goals were based on the Dietary Guidelines for Australian Adults, which promote eating a diverse diet that includes fruits, vegetables, grains, cereals, lean meat and fish while limiting the consumption of saturated fat, salt, alcohol and foods that contain added sugars [32]. The ManUp daily healthy eating goals were to: (1) eat two serves of fruit, (2) eat five serves of vegetables, (3) eat a serve of fish, (4) choose whole-grain bread instead of white bread, (5) choose low-fat dairy products, (6) have a soft drink- (soda-) free day, (7) have an alcohol-free day, (8) have an red-meat-free day, (9) have an unhealthysnack-free day, and (10) have a fast-food-free day. To promote dietary diversity, participants were not constrained to pre-selecting any specific ManUp healthy eating goals for the duration of a healthy eating challenge. Rather, participants were encouraged to achieve any of the ten ManUp healthy eating goals on a daily basis and in order to complete a challenge successfully the number of healthy eating goals needed to be achieved varied by the strength of the challenge selected (Table 2).

Differences in challenge strengths were designed to cater for varying levels of initial physical activity and nutrition behaviours, to generate confidence to achieve a realistic target for behaviour change and to provide the opportunity to progressively increase changes to physical activity and dietary habits. Concise information on the 'why', 'how', and 'where' for each physical activity and healthy eating goal was provided to participants. The 'why' focused on the health, social, or economic benefits of engaging in the activity or achieving the healthy eating goal. The 'how' provided tips on how to integrate the activity or healthy eating goal into the daily routine to overcome barriers associated with participation. The 'where' provided participants with local information in the Gladstone and Rockhampton areas on where to engage in the activity or purchase the food or healthy alternatives to unhealthy dietary choices.

\section{Intervention arms}

\section{IT-based intervention arm}

The IT-based intervention arm included access to the password-protected ManUp website. The website contained six sections that participants could navigate, including: My Profile, My Progress, My Mates, My Groups, My Weight, and Information Centre. The My Profile section summarized each participant's progress in their current challenges, allowed participants to record their progress towards any current challenges and post personal updates to their profile, schedule future activities, and displayed their groups and a list of their 'mates' (online friends on the website). The My Progress page allowed participants to examine their progress graphs towards their current challenges. The website allowed participants to search for and view mates on the My Mates section of the website. The My Groups section allowed participants to create a group and view the progress of groups they were part of. The My Weight section provided information on the benefits of achieving a healthy weight and allowed participants to record their height, weight and waist circumference. This information was used to provide automatically generated classifications of health risk based on waist circumference and body mass index (BMI). The Information Centre provided participants with summaries of information related to physical activity and healthy eating, as described in Table 1, and also information about the physical activity and healthy eating challenges.

The IT-based intervention arm promoted social support, and friendly competition among male peers (group-based challenges) as these have been reported as effective strategies to promote engagement in physical activity and healthy eating [12]. Social support was operationalised by allowing men to 'challenge' their mates to compete with them to achieve a goal either in a one-onone basis, or as part of a larger group. Men were also able to view the progress of their mates in any challenge they were enrolled in and to comment on their mates' My Profile page.

A mobile phone web application was developed as an additional tool to facilitate quick and convenient recording of progress towards the ManUp challenges, rather than as a platform to deliver educational material [21]. A mobile phone web application, rather than an "installed" application specific to a mobile platform (Apple, Android, Windows), was developed to maximize access to this additional self-monitoring tool. Any participant in the IT-based intervention arm who owned a mobile phone capable of accessing the internet had access to the mobile phone web application. The mobile phone web application allowed participants to enter their body weight, start a new ManUp physical activity or healthy eating challenge, record progress and view progress towards completing challenges. Both platforms were connected through protocols that enable data entry to be automatically synchronized between platforms on a frequently scheduled period. 


\section{Print-based intervention arm}

Participants in the print-based group received a hard copy booklet that provided the same educational materials and ManUp challenges as those received by participants in the IT-based intervention. Participants in the print-based group were not provided with information on their peers in this group. The print-based booklet also included log sheets that could be used to monitor their progress and/or successful completion of any of the ManUp physical activity or healthy eating challenges.

\section{Measures}

All participants completed an online questionnaire at baseline and completed follow-up online questionnaires at 3 months and 9 months after they began the intervention. A randomly selected sub-sample $(n=91,61$ from IT-based intervention arm, 30 from print-based intervention arm) of participants also attended one of the trial centres (CQUniversity Gladstone or Rockhampton Campus) for an in-person assessment at each time point. During in-person assessments, participants completed the online survey, received an accelerometer for the objective measurement of physical activity, and had their height, weight and waist circumference measured by a trained research assistant.

\section{Demographic characteristics}

Participants self-reported the following demographic characteristics: age, employment status, level of employment, industry of employment, number of hours of work per week, household income, years of education, number of children (aged $<18$ years) living in the household, living situation, and presence of any chronic diseases diagnosed by a doctor.

\section{Perceived health status}

Participants reported on several aspects of perceived health status that have been identified as risk factors for health behaviours and chronic disease [16,33]. Two items were used to assess risk perception in relation to physical activity and body weight, consistent with previous research [16]. The items are "I believe that I am doing enough exercise/physical activity to achieve health benefits" and "I believe that my current body weight is a risk to my health." Responses were measured on a five-point Likert type scale from strongly agree to strongly disagree [16]. The self-rated health item from the Healthy Days Module of the Behavioral Risk Factor Surveillance Survey (BRFSS) was used to assess self-rated health, and response options were excellent, very good, good, fair or poor [34].

\section{Weight}

All participants self-reported current height $(\mathrm{cm})$, weight $(\mathrm{kg})$, and waist circumference $(\mathrm{cm})$ during the online survey. For those individuals selected for in-person assessments, these measures are also measured by project staff. During in-person assessments, participant height to the nearest $0.1 \mathrm{~cm}$ (PE087, Mentone Educational, Victoria, Australia), weight to the nearest $0.1 \mathrm{~kg}$ (Tanita BF-681, Tanita Corp., Tokyo, Japan) and waist circumference to the nearest $0.1 \mathrm{~cm}$ (Lufkin Executive W606PM) are measured at each assessment point, in triplicate. Participants are asked to wear light clothing and to remove shoes prior to the assessment. Waist circumference is measured horizontally at the umbilicus, after normal expiration, by a trained research assistant.

\section{Activity related behaviours}

Physical activity was assessed in all participants using the Active Australia Questionnaire, which assesses the frequency and duration of transport and recreational walking, moderate and vigorous intensity physical activity [35]. The Active Australia Questionnaire has demonstrated acceptable levels of test re-test reliability and validity in the Australian adult population, and has been identified as a useful measure to detect interventionrelated change in physical activity behaviours [36-39]. Participants $(n=91)$ who selected to attend in-person measurement sessions were fitted with an ActiGraph GT3X (ActiGraph, Pensacola, FL) accelerometer to provide objective measures of activity behaviours over a five-day period. Participants were instructed to wear the ActiGraph, mounted on an elastic belt around the waist with the unit positioned over the right hip during all waking, non-contact activities (thus excluding activities like rugby league or rugby union) and non-water-based activities. The ActiGraph monitors were set to record steps, inclination, and acceleration counts in tri-axial mode, using a 10-second epoch. Accelerometer data was analysed using the MeterPlus program [40] in 10 second epochs using previously reported cut-points for sedentary, light and moderate-to-vigorous intensity physical activity [41]. Non wear time was assessed using a minimum of 60 minutes of consecutive zero counts allowing up to a 2 minute tolerance of non-zero counts. A minimum of 10 hours per day of wear time on at least four days was required to be included in analysis.

Self-reported duration of sitting in occupational settings over the previous seven days was assessed using two items. Adapted from an existing measure of occupational sitting [5,42], the first item asked participants to report the amount of time sitting at work during meetings, lunch and at their desk. Using the same recall period the second item asked participants to report the amount of time spent driving at work. Both items asked participants to report the duration of sitting in hours and minutes. Duration of sitting in leisure time was assessed using items adapted from a existing measure of nine leisure- 
time sedentary behaviours with demonstrated acceptable test-retest reliability [43]. The sedentary behaviours that were assessed included computer use, hobbies, television viewing, sitting and socializing, reading, sitting or lying down while listening to music, talking on the telephone, going for a recreational drive, and relaxing, thinking and resting [43]. These items were modified to explicitly ask about time spent sitting while performing each behaviour, instead of time spent engaged in each behaviour.

\section{Nutrition behaviours}

Nutrition behaviours related to the healthy eating guidelines for Australian adults [32] were assessed using 19 items adapted from the National Health Survey Australia and the Monitoring Food Habits Questionnaire $[44,45]$. The number of daily serves of fruit and vegetables usually consumed in the previous week was assessed using two separate items, based on those used in the National Health Survey [44]; possible response options were: one serve or less; two to three serves; four to five serves; six or more; and don't consume this food. The number of times in the previous week that red meat, fish, meat products (sausages, salami, meat pies, etc.), cooked cereals, bread, soft drink, chips, takeaway foods, and sweet or savoury foods were consumed was assessed using a nine-point scale from rarely/never to more than seven times per week, each item also included a "don't consume this food" response option. The volume of milk consumed each day and the type of milk (whole milk, reduced fat, soy milk, condensed milk) was assessed using two separate items [44].

\section{Health literacy}

Health literacy can be defined as the acquisition of a set of skills and knowledge that can be applied to change behaviours and improve health $[22,46]$. The five physical activity awareness items from the Active Australia Questionnaire were used to assess health literacy related to physical activity [35]. These items assess awareness related to the health benefits of physical activity that include the minimum amount of physical activity required for health benefit, the appropriate intensity of physical activity to achieve benefit, and the pattern in which physical activity can be accumulated to receive health benefits [35]. The 28-item Nutritional Literacy Survey was used to assess health literacy related to healthy eating [47]. This instrument presents sentences to the respondent that contain one or more words removed from the sentence, the respondent is provided with four possible response options and asked to select the response that best fits the sentence [47]. Topics covered include food types that promote heart health, fat and cholesterol contents of food, and portion size [47].

\section{IT platform usage}

Both the website and mobile platforms allow monitoring of the number of times a participant has logged in, the number and date of entries made, and the number of challenges created and completed. The website platform also permits monitoring of which educational resource pages have been viewed by participants. These measures describe the level of participant engagement with the intervention components.

\section{Sample size}

The study was powered to detect a 60-minute increase in moderate-to-vigorous intensity physical activity using a 0.05 alpha, with a power level of $90 \%$. Based on these factors, it was estimated that 197 participants would be required [48]. Typical dropout rates in IT based interventions are approximately 30\% [16]. Given the difficulty in engaging and retaining the target population (middle aged males) the estimated sample size was increased to account for a $45 \%$ dropout rate. Methods described by Hsieh to account for the loss of power associated with the 2:1 group allocation [49], the estimated sample size was further inflated using a variance inflation factor of $(\mathrm{VIF}=1.125)$ resulting in a total estimated sample size of 321; 107 allocated to the print-based group and 214 allocated to the IT-based group.

\section{Analysis}

Comparison of sample baseline characteristics between intervention arms were examined using chi-square tests for proportions, and either linear, gamma or Poisson generalized linear models for continuous or count data. Examination of change in outcomes will be based on group allocation and the intention-to-treat principle. Linear mixed models and generalized estimating equations will be used to compare intervention groups across time points [50]. Statistical significance was set at a $p$-value of 0.05 .

\section{Baseline characteristics of the sample}

A total of 327 males contacted the research team about participation in the study, 10 males withdrew from the study prior to randomization to an intervention arm (Figure 1). The primary reason for not continuing participation in the study was being no longer interested in participation after having details of the study explained to them. Following allocation to an intervention arm, nine participants in the IT-based intervention arm and seven participants in the print-based intervention arm did not complete a baseline assessment and were 


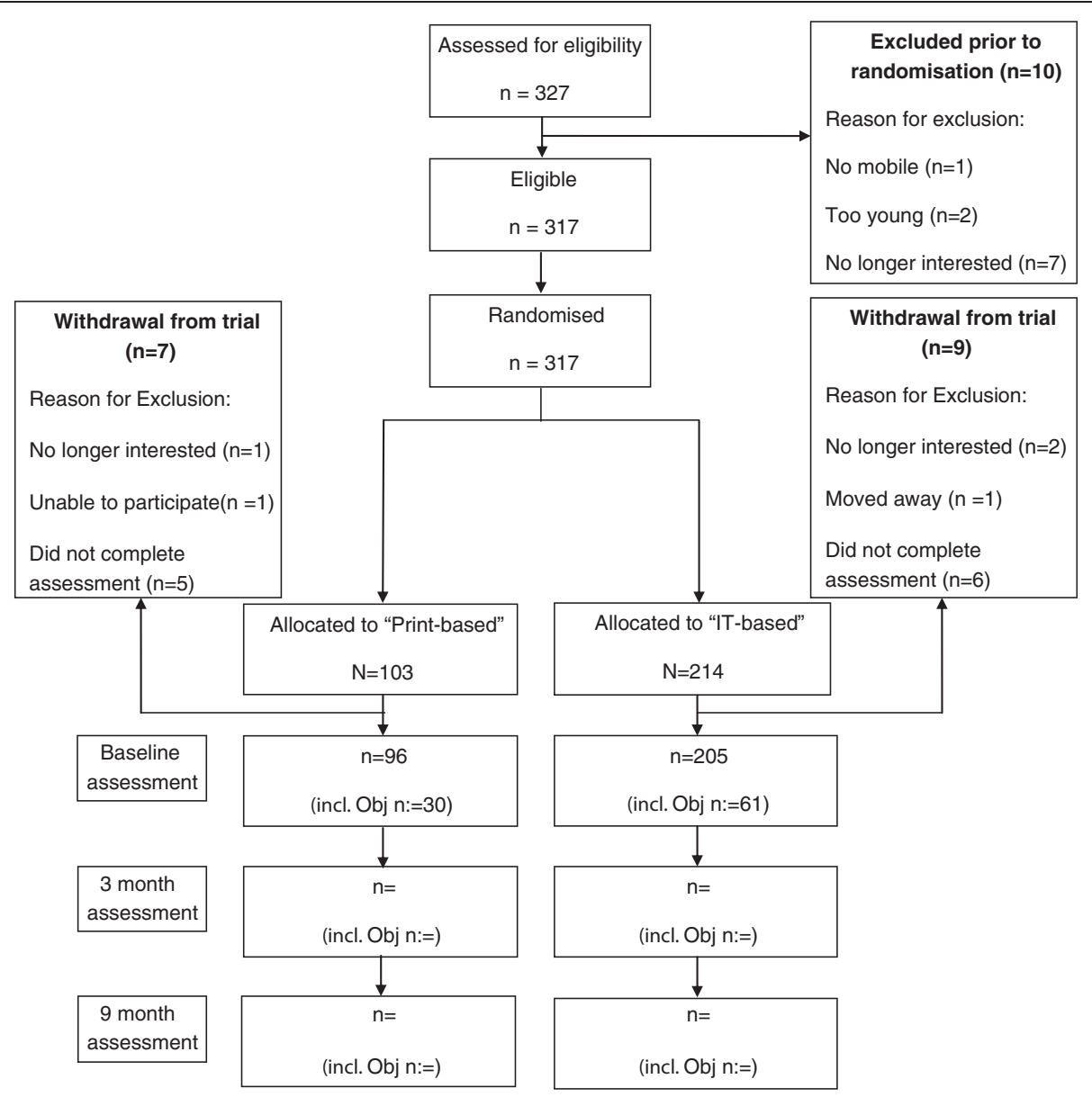

Figure 1 Flowchart describing the progress of participants through trial phases. Obj - Objective or 'in person' measurement completed with randomly selected sub-sample of participants.

withdrawn from the trial (Figure 1). Participants were asked how they learned about the existence of the ManUp project (for which they could report multiple methods); responses were classified into the following categories 1) project specific advertisements and promotional materials $(n=152), 2)$ word of mouth $(n=87), 3)$ information provided at their workplace $(\mathrm{n}=75), 4)$ direct contact with project staff $(n=49)$, and 5) not specified $(n=1)$.

Participant socio-demographic characteristics at baseline are provided in Table 3. The average age of IT-based and print-based intervention arms was similar (44.2 vs. $43.8 p=.656)$. In both intervention arms, the majority of participants were classified as working in professional occupations; there were no significant differences between intervention arms in the proportion of participants employed in professional, white collar, blue collar or other occupation categories $(p=.639)$. All participants owned a mobile phone, however only 151 (73.2\%) of those in the IT-based intervention arm owned a mobile phone that was capable of accessing the internet. The average BMI (calculated from self-reported height and weight) was 30.9 and 30.4 in the IT-based and printbased intervention arms respectively $(p=.434)$, when classifying BMI in established BMI categories, over $85 \%$ of participants in both intervention arms were classified as either overweight or obese (Table 3). The IT-based and print-based intervention arms did not significantly differ on average for the in-person measured BMI ( 29.8 vs. $30.2, p=.712$ ) and over $80 \%$ of participants were classified as overweight or obese when using in-person measured BMI. There were no significant between-group differences for any socio-demographic variable examined in Table 3.

Table 4 shows that there were no significant betweengroup differences in: self-reported minutes or sessions of physical activity; sitting during leisure time; sitting at work; or minutes of objectively determined minutes of sedentary, light and moderate-to-vigorous intensity physical activity. When examining physical activity literacy, the 
Table 3 Baseline comparisons of socio-demographic and anthropometric characteristics between ManUp intervention groups $(n=301)$

\begin{tabular}{|c|c|c|c|c|c|}
\hline \multirow[b]{2}{*}{ Variable } & \multicolumn{2}{|c|}{ Print-based } & \multicolumn{2}{|c|}{ IT-based } & \multirow[b]{2}{*}{$\mathrm{p}$} \\
\hline & $\mathbf{N}$ & $\%, M(S E)$ & $\mathbf{N}$ & $\%, M(S E)$ & \\
\hline Age & 96 & $43.8(.6)$ & 205 & $44.2(.4)$ & .656 \\
\hline \multicolumn{6}{|l|}{ Occupational Classification } \\
\hline$\%$ Professional & 52 & $54.2 \%$ & 118 & $57.6 \%$ & \\
\hline \% White Collar & 8 & $8.3 \%$ & 16 & $7.8 \%$ & \\
\hline \% Blue Collar & 23 & $24.0 \%$ & 37 & $18.0 \%$ & \\
\hline$\%$ Other $^{\mathrm{b}}$ & 13 & $13.5 \%$ & 34 & $16.6 \%$ & 639 \\
\hline \multicolumn{6}{|l|}{ Education Level } \\
\hline \% Secondary School or Less & 20 & $20.8 \%$ & 45 & $22.0 \%$ & \\
\hline$\%$ TAFE & 25 & $26.0 \%$ & 61 & $29.8 \%$ & \\
\hline$\%$ University & 51 & $53.1 \%$ & 99 & $48.3 \%$ & .719 \\
\hline Self-Report Weight & 96 & $96.3(2.0)$ & 205 & $98.3(1.4)$ & .389 \\
\hline Self-Reported BMI & 96 & $30.4(.5)$ & 205 & $30.9(.4)$ & .434 \\
\hline \multicolumn{6}{|l|}{ Self-Reported BMI Classification } \\
\hline$\%$ Healthy Weight & 12 & $12.8 \%$ & 19 & $9.3 \%$ & \\
\hline$\%$ Overweight & 40 & $42.6 \%$ & 85 & $41.5 \%$ & \\
\hline$\%$ Obese & 42 & $44.7 \%$ & 101 & $49.3 \%$ & .588 \\
\hline Self-Reported Waist Circumference & 96 & $98.9(2.0)$ & 205 & $99.6(1.4)$ & .782 \\
\hline \multicolumn{6}{|c|}{ Self-Reported Waist Circumference Classification } \\
\hline$\%$ Healthy & 33 & $34.4 \%$ & 59 & $28.8 \%$ & \\
\hline \% Risky & 28 & $29.2 \%$ & 50 & $24.4 \%$ & \\
\hline \% High Risk & 35 & $36.5 \%$ & 96 & $46.8 \%$ & .239 \\
\hline \multicolumn{6}{|l|}{ Self-Rated Health Classification } \\
\hline$\%$ Fair or Poor & 25 & $26.0 \%$ & 73 & $35.6 \%$ & \\
\hline$\%$ Good & 37 & $38.5 \%$ & 83 & $40.5 \%$ & \\
\hline$\%$ Very Good or Excellent & 34 & $35.4 \%$ & 49 & $23.9 \%$ & .080 \\
\hline Measured Weight ${ }^{a}$ & 30 & $94.7(3.1)$ & 61 & $93.3(2.2)$ & .719 \\
\hline Measured BMI ${ }^{a}$ & 30 & $30.2(.9)$ & 61 & $29.8(.6)$ & .712 \\
\hline \multicolumn{6}{|l|}{ Measured BMI Classification ${ }^{a}$} \\
\hline$\%$ Healthy Weight & 3 & $10.0 \%$ & 10 & $16.4 \%$ & \\
\hline$\%$ Overweight & 14 & $46.7 \%$ & 26 & $42.6 \%$ & \\
\hline$\%$ Obese & 13 & $43.3 \%$ & 25 & $41.0 \%$ & .713 \\
\hline Measured Waist Circumference ${ }^{a}$ & 30 & $101.9(2.3)$ & 61 & $102.0(1.6)$ & .968 \\
\hline \multicolumn{6}{|c|}{ Measured Waist Circumference Classification ${ }^{a}$} \\
\hline$\%$ Healthy & 6 & $20.0 \%$ & 15 & $24.6 \%$ & \\
\hline \% Risky & 11 & $36.7 \%$ & 19 & $31.1 \%$ & \\
\hline$\%$ High Risk & 13 & $43.3 \%$ & 27 & $44.3 \%$ & .830 \\
\hline
\end{tabular}

Note. ${ }^{\text {a }}$ A randomly selected sub-sample $(n=91)$ of participants completed in person assessments of height, weight, waist circumference and physical activity (ActiGraph) these are referred to as "measured" outcomes.

${ }^{b}$ Other category includes participants who were retired, students, unemployed or a pensioner.

only significant between-group difference was that a lower proportion of participants in the IT-based intervention arm agreed that 30 minutes each day is enough to improve health compared to the print-based intervention arm $(70.2 \%$ vs. $82.3 \%, p=.026)$. No significant differences were observed between the IT-based intervention arm and the print-based intervention arm on the proportion of participants who reported eating at least two serves of 
Table 4 Baseline comparisons of physical activity and dietary behaviours between ManUp intervention groups $(n=301)^{a}$

\begin{tabular}{|c|c|c|c|c|c|}
\hline & \multicolumn{2}{|r|}{ Print-based } & \multicolumn{2}{|r|}{ IT-based } & \multirow[b]{2}{*}{$\mathrm{p}$} \\
\hline & $\mathbf{N}$ & $\begin{array}{l}\%, M(S E) \text { or median } \\
\left(1^{\text {st }} \text { and } 3^{\text {rd }} \text { quartile }\right)\end{array}$ & $\mathbf{N}$ & $\begin{array}{l}\%, M(S E) \text { or median } \\
\left(1^{\text {st }} \text { and } 3^{\text {rd }} \text { quartile }\right)\end{array}$ & \\
\hline \multicolumn{6}{|l|}{ Self-report physical activity behaviours } \\
\hline Weekly minutes of physical activity & 96 & $277.9(34.0)$ & 205 & $286.1(23.3)$ & .843 \\
\hline Weekly session of physical activity & 96 & $4(1,8)$ & 205 & $4(1,7)$ & .892 \\
\hline \multicolumn{6}{|l|}{ Physical activity classification } \\
\hline$\%$ None & 19 & $19.8 \%$ & 39 & $19.0 \%$ & \\
\hline$\%$ Insufficient & 36 & $37.5 \%$ & 88 & $42.9 \%$ & \\
\hline$\%$ Sufficient & 41 & $42.7 \%$ & 78 & $38.0 \%$ & .655 \\
\hline Daily minutes of sitting outside of work & 96 & $520.1(27.2)$ & 205 & $492.9(18.6)$ & .409 \\
\hline Daily minutes of sitting at work & 96 & $411.0(26.1)$ & 205 & $452.4(17.9)$ & .191 \\
\hline \multicolumn{6}{|l|}{ Objective Physical Activity Behaviours ${ }^{\mathrm{a}}$} \\
\hline Daily minutes of sedentary behaviour & 25 & $631.0(18.5)$ & 52 & $670.6(12.8)$ & .079 \\
\hline Daily minutes of light intensity physical activity & 25 & $184.9(8.4)$ & 52 & $182.1(5.8)$ & .786 \\
\hline Daily minutes of moderate-to-vigorous intensity physical activity & 25 & $46.7(4.9)$ & 52 & $44.8(3.2)$ & .751 \\
\hline \multicolumn{6}{|l|}{ Physical Activity Literacy (\% Agree) } \\
\hline$>30$ minutes/day improves health & 79 & $82.3 \%$ & 144 & $70.2 \%$ & .026 \\
\hline 30 minutes brisk walking improves health & 79 & $82.3 \%$ & 153 & $74.6 \%$ & .141 \\
\hline 20 minutes of Vigorous activity 3 times a week is essential & 54 & $56.3 \%$ & 139 & $67.8 \%$ & .051 \\
\hline 10 minute blocks of activity are okay & 52 & $54.2 \%$ & 106 & $51.7 \%$ & .690 \\
\hline Moderate activity can improve health & 87 & $90.6 \%$ & 177 & $86.3 \%$ & .291 \\
\hline \multicolumn{6}{|l|}{ Dietary Habits } \\
\hline \# Serves vegetables/day & 96 & $2(1,2)$ & 205 & $2(1,3)$ & .793 \\
\hline \# Serves fruit/day & 96 & $1(1,2)$ & 205 & $1(1,2)$ & .735 \\
\hline \# Serves of red meat last week & 96 & $4(3,6)$ & 205 & $4(3,5)$ & .017 \\
\hline \# Soft drinks last week & 96 & $2(0,3)$ & 205 & $1(0,4)$ & .783 \\
\hline \# Times fast food/takeaway & 96 & $2(0,2.75)$ & 205 & $(1(.5,2)$ & .339 \\
\hline Ave. Serves of Alcohol on a drinking day & 96 & $2.78(.3)$ & 205 & $2.68(.2)$ & .761 \\
\hline \multicolumn{6}{|l|}{ \# Days of Harmful Drinking } \\
\hline$\% 0$ days & 45 & $46.9 \%$ & 102 & $49.8 \%$ & \\
\hline$\% 1-2$ days & 29 & $30.2 \%$ & 60 & $29.3 \%$ & \\
\hline$\% \geq 3$ days & 22 & $22.9 \%$ & 43 & $21.0 \%$ & .885 \\
\hline \multicolumn{6}{|l|}{ Bread Type } \\
\hline$\%$ White & 40 & $42.6 \%$ & 62 & $31.3 \%$ & \\
\hline \% Grain & 53 & $56.4 \%$ & 133 & $67.2 \%$ & \\
\hline \% Don't Eat Bread & 1 & $1.1 \%$ & 3 & $1.5 \%$ & .168 \\
\hline \multicolumn{6}{|l|}{ Milk Type } \\
\hline$\%$ Full Cream & 37 & $38.5 \%$ & 80 & $39.0 \%$ & \\
\hline$\%$ Low Fat & 50 & $52.1 \%$ & 102 & $49.8 \%$ & \\
\hline \% Soy/Condensed & 2 & $2.1 \%$ & 5 & $2.4 \%$ & \\
\hline \% Don't Drink Milk & 2 & $2.1 \%$ & 5 & $2.4 \%$ & \\
\hline$\%$ Other & 5 & $5.2 \%$ & 13 & $6.3 \%$ & .991 \\
\hline Nutritional Literacy & 96 & $25(24,26)$ & 205 & $26(24,27)$ & .656 \\
\hline
\end{tabular}

Note: ${ }^{a}$ A randomly selected sub-sample $(n=91)$ of participants completed in person assessments of height, weight, waist circumference and physical activity (ActiGraph). Variation in sample size is due to participants not fulfilling wear time criteria and were excluded from analysis of objective measures of physical activity. 
fruit per day (38.5\% vs. $35.4 \%, p=.602)$ and five or more serves of vegetables per day (5.9\% vs. $5.2 \%, p=.821)$. The print-based intervention arm consumed a higher number of serves of red meat compared to the IT-based intervention $\operatorname{arm}(p=.017)$. No other significant differences were observed in nutrition behaviours or levels of nutrition literacy between the intervention groups.

\section{Discussion}

This paper describes the intervention design, study proto$\mathrm{col}$ and baseline characteristics of a sample of middle aged males who took part in the ManUp Study. This study was designed in an attempt to address some of the key issues associated with conducting research in the middle-aged male population. These issues include specifically designing the intervention with the needs and preferences of males in mind to increase intervention appeal, and focusing on behavioural risk factors most relevant to men. The study protocol will allow the efficacy of an IT-based delivery of the intervention to be compared to a print-based delivery mode. If the IT-based delivery mode is found to be efficacious, it will provide the foundation for similar interventions that take advantage of the increased reach of IT-based interventions to be developed for this population group. Within the IT-based intervention arm, it will be possible to determine the presence of any relationships between engagement with the platform and change in outcomes. This will provide much needed information in this area as reviews of such interventions have identified that a low proportion of males engage in these interventions $[12,19]$. The ManUp Study will also collect information on the components of the IT-based intervention that are utilized, and this information can be used to refine the strategies adopted in future IT-based interventions by focusing attention on those that participants use most frequently.

Males are frequently reported to have high levels of anthropometric and behavioural risk factors of poor health, and this is the underlying rationale for the current study $[2,3,8]$. Baseline characteristics of the ManUp Study participants indicate, that like many males, they are generally overweight or obese, have poor nutrition behaviours and have a low level of total physical activity. Over $85 \%$ and $80 \%$ of participants are classified as overweight or obese when using self-reported and measured BMI respectively; this is higher than levels of self-reported overweight or obesity in Queensland and Australian adult males [3,8,51]. The proportion of participants who self-reported physical activity at a level sufficient to meet guidelines in printand IT-based intervention arms (42.7\% and 38.0\%) is lower than that observed in population-based samples covering similar geographical areas (52.8\%) [6]. Furthermore, in comparison to other available data [52], the current sample spends more time in sedentary behaviour and less time in light intensity physical activity. The average nutrition behaviours of the study sample were below that recommended for Australian adults, and the proportion of the sample who achieved the minimum guidelines for fruit and vegetables was lower than that reported for other Australian males [8].

Levels of physical activity literacy in the current sample were lower than those previously reported in Australian males [53], particularly for awareness that accumulating physical activity in 10-minute blocks can still provide health benefits. Over a decade has passed and significant financial investment has been directed toward the promotion of physical activity in Australia and the levels of physical activity literacy in the current sample are lower than those reported in 1999 [53]. While this is concerning from a health promotion perspective, it also provides a unique opportunity to intervene and attempt to address the issue in the current sample of participants to inform subsequent promotion efforts. In contrast, levels of nutrition literacy were relatively high with the median score of both intervention groups close to the maximum level and comparable to levels reported in the sample the instrument was developed in [47]. This is a positive finding in the current study, and somewhat unexpected given that males in our formative study reported confusion around nutritional messages [20].

Subsequent phases of the ManUp Study will evaluate changes in physical activity, nutrition behaviours, and health literacy of those topics over the intervention period, and will compare the efficacy of the two intervention arms to change these outcomes.

\section{Competing interests}

The authors declare that they have no competing interests.

\section{Authors' contributions}

MJD drafted the manuscript and completed statistical analysis. AM, RT, MK and $\mathrm{HD}$ developed the IT platform. MJD, CV, CH and ME were responsible for participant recruitment and overseeing study implementation. All authors contributed to the design of the overall study, development of intervention materials, read, edited and approved the final manuscript.

\section{Acknowledgements}

Queensland Health provided funding to conduct this project. CV is supported by a National Health and Medical Research Council of Australia (\#519778) and National Heart Foundation of Australia (\#PH 07B 3303) postdoctoral research fellowship.

\section{Author details}

${ }^{1}$ Central Queensland University, Institute for Health and Social Science Research, Centre for Physical Activity Studies, Bruce Highway, Rockhampton, QLD 4700, Australia. ${ }^{2}$ Kansas State University, Department of Human Nutrition, , Manhattan, Kansas 66506, United States. ${ }^{3}$ University of British Columbia, School of Health and Exercise Sciences, Kelowna, British Columbia V1V 1V7, Canada. ${ }^{4} \mathrm{CSIRO}$, The Australian eHealth Research Centre, ICT Centre, Level 5, UQ Health Sciences Building 901/16, Royal Brisbane and Women's Hospital, Herston QLD, 4029, Australia. ${ }^{5}$ University of Western Sydney, School of Science and Health, Locked Bag 1797, Penrith NSW 2751, Australia. 6University of Western Sydney, School of Computing, Engineering and Mathematics, Tele-Health Research and Innovation Laboratory, Narellan Road, Campbelltown, NSW 2560, Australia. ${ }^{7}$ CSIRO, Food and Nutritional Sciences, PO Box 10041, Adelaide, BC 5000, Australia. ${ }^{8}$ Central Queensland University, 
Boundary Road, Mackay, QLD 4740, Australia. ${ }^{9}$ University of Alberta, Faculty of Physical Education and Recreation, Edmonton, Alberta, T6G 2H9, Canada.

Received: 27 June 2012 Accepted: 2 August 2012

Published: 15 August 2012

\section{References}

1. Wilkins D: Introduction. Belgium: European Men's Health Forum (EMHF0); 2009.

2. Department of Health and Aging: Building on the strengths of Australian males - healthy routines. In National Male Health Policy. Edited by Australian Government. Canberra: Canberra; 2010.

3. Australian Bureau of Statistics: Men's Health: Australian Social Trends: 4102. Canberra: Australian Bureau of Statistics; 2010.

4. Thorp AA, Healy GN, Owen N, Salmon J, Ball K, Shaw JE, Zimmet PZ, Dunstan DW: Deleterious associations of sitting time and television viewing time with cardiometabolic risk biomarkers: Australian diabetes, obesity and lifestyle (AusDiab) study 2004-2005. Diabetes care 2010, 33:327-334.

5. Duncan MJ, Badland HM, Mummery WK: Physical activity levels by occupational category in non-metropolitan Australian adults. J Phys Act Health 2010, 7:718-723.

6. Vandelanotte C, Duncan MJ, Caperchione C, Hanley C, Mummery WK: Physical activity trends in Queensland (2002 to 2008): are women becoming more active than men? Aust N Z J Public Health 2010, 34:248-254.

7. DiSipio T, Rogers C, Newman B, Whiteman D, Eakin E, Fritschi L, Aitken J: The Queensland cancer risk study: behavioural risk factor results. Aust $N$ Z J Public Health 2006, 30:375-382.

8. Australian Institute of Health and Welfare: The health of Australia's males. Canberra: AlHW: In: PHE 141; 2011.

9. Australian Bureau of Statistics: 4832.0.55.001 - Alcohol Consumption in Australia: A Snapshot, 2004-05. Canberra: Australian Bureau of Statistics; 2006.

10. Queensland Health: The Health of Queenslanders 2010. Brisbane: Third Report of the Chief Health Officer; 2010.

11. Waters LA, Galichet B, Owen N, Eakin E: Who participates in physical activity intervention trials? J Phys Act Health, 8:85.

12. George ES, Kolt GS, Duncan MJ, Caperchione CM, Mummery WK, Vandelanotte C, Taylor P, Noakes M: A review of the effectiveness of physical activity interventions for adult males. Sports Med 2012, 42:281-300.

13. Burton NW, Walsh A, Brown WJ: It just doesn't speak to me: mid-aged men's reactions to '10,000 Steps a Day'. Health Promot J Austr 2008, 19:52-59.

14. Morgan PJ, Collins CE, Plotnikoff RC, Cook AT, Berthon B, Mitchell S, Callister $R$ : Efficacy of a workplace-based weight loss program for overweight male shift workers: the workplace power. (Preventing Obesity Without Eating like a Rabbit) randomized controlled trial. Prev Med, 52:317-325.

15. Morgan PJ, Warren JM, Lubans DR, Collins CE, Callister R: Engaging men in weight loss: Experiences of men who participated in the male only SHED-IT pilot study. Obesity Research \& Clinical Practice 2011, 5:e239-e248.

16. Vandelanotte C, Spathonis KM, Eakin EG, Owen N: Website-delivered physical activity interventions a review of the literature. Am J Prev Med 2007, 33:54-64.

17. Kirwan M, Duncan MJ, Vandelanotte C, Mummery WK: Using smartphone technology to monitor physical activity in the 10,000 steps program: A matched case controlled trial. J Med Internet Res 2012, 14:e55.

18. Neve M, Morgan PJ, Jones PR, Collins CE: Effectiveness of web-based interventions in achieving weight loss and weight loss maintenance in overweight and obese adults: a systematic review with meta-analysis. Obes Rev, 11:306-321.

19. Davies CA, Spence JC, Vandelanotte C, Caperchione CM, Mummery WK: Meta-analysis of internet-delivered interventions to increase physical activity levels. Int I Behav Nutr Phys Act 2012, 9:52.

20. Caperchione CM, Vandelanotte C, Kolt GS, Duncan M, Ellison M, George E, Mummery WK: What a man wants: Understanding the challenges and motivations to physical activity participation and healthy eating in middle-aged Australian men. Am J Mens Health 2012.

21. Vandelanotte C, Caperchione C, Ellison M, George E, Maeder A, Kolt G, Duncan M, Karunanithi M, Noakes M, Hooker C, et al: What kind of website and mobile phone delivered physical activity and nutrition interventions do middle-aged men want? J Health Commun, In Press.

22. Nutbeam D: The evolving concept of health literacy. Soc Sci Med 2008, 67:2072-2078

23. Webb TL, Joseph J, Yardley L, Michie S: Using the internet to promote health behavior change: A systematic review and meta-analysis of the impact of theoretical basis, use of behavior change techniques, and mode of delivery on efficacy. J Med Internet Res 2010, 12(1):e4.

24. Marcus BH, Lewis BA, Williams DM, Dunsiger S, Jakicic JM, Whiteley JA, Albrecht AE, Napolitano MA, Bock BC, Tate DF, et al: A comparison of Internet and print-based physical activity interventions. Arch Intern Med 2007, 167:944-949

25. Short CE, James EL, Plotnikoff RC, Girgis A: Efficacy of tailored-print interventions to promote physical activity: a systematic review of randomised trials. Int J Behav Nutr Phys Act 2011, 8:113.

26. The Sports Medicine Australia pre-exercise screening system. 2005. http://sma. org.au/wp-content/uploads/2009/05/new_pre_screening.pdf.

27. Cullen KW, Baranowski T, Smith SP: Using goal setting as a strategy for dietary behavior change. J Am Diet Assoc 2001, 101:562-566.

28. Bandura A: Social Cognitive Theory of self-regulation. Organi Behav Hum Decis 1991, 50:248-287.

29. Maes S, Karoly P: Self-Regulation assessment and intervention in physical health and illness: A review. Applied Psychology: An international review 2005, 54:267-299.

30. Abraham C, Michie S: A taxonomy of behavior change techniques used in interventions. Health psychology: official journal of the Division of Health Psychology, American Psychological Association 2008, 27:379-387.

31. Australian Bureau of Statistics: Participation in Sport and Physical Recreation, Australia. 4177. Canberra: Australian Bureau of Statistics; 2010.

32. National Health: Medical Research Council: Dietary Guidelines for Australian Adults. Canberra: National Health \& Medical Research Council; 2003.

33. Tsai J, Ford ES, Li C, Zhao G, Pearson WS, Balluz LS: Multiple healthy behaviors and optimal self-rated health: findings from the,2007. Behavioral Risk factor surveillance system survey. Prev Med 2007, 51:268-274.

34. Centers for Disease Control and Prevention: Measuring Healthy Days. In Measuring Healthy Days. Edited by CDC. Atlanta, Georgia: CDC; 2000.

35. Australian Institute of Health and Welfare (AlHW): A guide and manual for implementation, analysis and reporting. Canberra: AlHW: he Active Australia survey; 2003

36. Brown W, Bauman A, Chey T, Trost S, Mummery K: Comparison of surveys used to measure physical activity. Aust N Z J Public Health 2004, 28:128-134.

37. Timperio A, Salmon J, Bull F, Rosenburg M: Book validation of adult physical activity questions for use in Australia population surveys (Editor ed.^eds.). In Validation of adult physical activity questions for use in Australia population surveys. City: Department of Health and Ageing; 2002. unpublished report.

38. Brown WJ, Bauman A, Trost S, Mummery WK, Owen N: Test-retest reliability of four physical activity measures used in population surveys. J Sci Med Sport 2004, 7:205-215.

39. Reeves MM, Marshall AL, Owen N, Winkler EA, Eakin EG: Measuring physical activity change in broad-reach intervention trials. J Phys Act Health 2010, 7:194-202.

40. Santech Inc: MeterPlus version 4.3.: http://www.meterplussoftware.com.

41. Healy GN, Matthews CE, Dunstan DW, Winkler EA, Owen N: Sedentary time and cardio-metabolic biomarkers in US adults: NHANES 2003-06. European heart journal 2011, 32(5):590-597.

42. Mummery WK, Schofield GM, Steele R, Eakin EG, Brown WJ: Occupational sitting time and overweight and obesity in Australian workers. Am J Prev Med 2005, 29(2):91-97.

43. Salmon J, Owen N, Crawford D, Bauman A, Sallis JF: Physical activity and sedentary behavior: a population-based study of barriers, enjoyment, and preference. Health psychology: official journal of the Division of Health Psychology, American Psychological Association 2003, 22(2):178-188.

44. Australian Bureau of Statistics: National Health Survey Questionnaire 2004-05 4363.0.55.001. In: National Health Survey 2005 Users' Guide. Canberra: Australian Bureau of Statistics; 2005

45. Marks GC, Webb K, Rutishauser IHE, Riley M: Monitoring food habits in the Australian population using short questions. In: National Food and Nutrition Monitoring and Surveillance Project. Commonwealth Department of Health and Aged Care; 2001 
46. WHO Commission on the Social Determinants of Health: Achieving health equity: From root causes to fair outcomes. Geneva: World Health Organisation; 2007.

47. Diamond JJ: Development of a reliable and construct valid measure of nutritional literacy in adults. Nutr J 2007, 6:5.

48. Wittes J: Sample size calculations for randomized controlled trials. Epidemiol Rev 2002, 24(1):39-53.

49. Hsieh FY, Lavori PW, Cohen HJ, Feussner JR: An overview of variance inflation factors for sample-size calculation. Eval Health Prof 2003, 26(3):239-257.

50. Twisk J, de Vente W: Attrition in longitudinal studies. How to deal with missing data. J Clin Epidemiol 2002, 55(4):329-337.

51. Queensland Health: Overweight and obesity, Queensland. In: Self reported health status 2011 and child health status 2011. Brisbane: Queensland Health; 2011.

52. Healy GN, Dunstan DW, Salmon J, Cerin E, Shaw JE, Zimmet PZ, Owen N: Objectively measured light-intensity physical activity is independently associated with 2-h plasma glucose. Diabetes Care 2007, 30(6):1384-1389.

53. Armstong T, Bauman A, Davies J: Physical activity patterns of Australian adults. Results of the 1999 National Physical Activity Survey. Canberra: Australian Institute of Health and Welfare; 2000.

doi:10.1186/1471-2458-12-656

Cite this article as: Duncan et al:: Effectiveness of a website and mobile phone based physical activity and nutrition intervention for middleaged males: Trial protocol and baseline findings of the ManUp Study. BMC Public Health 2012 12:656.

\section{Submit your next manuscript to BioMed Central and take full advantage of:}

- Convenient online submission

- Thorough peer review

- No space constraints or color figure charges

- Immediate publication on acceptance

- Inclusion in PubMed, CAS, Scopus and Google Scholar

- Research which is freely available for redistribution 